\section{ADVANCE CARE PLANNING IN DEMENTIA: GREAT IN THEORY, A CHALLENGE IN PRACTICE}

10.1136/bmjspcare-2011-000053.56

L Robinson, C Dickinson, C Bamford, C Exley, J Hughes Newcastle Unversity, Newcastle Upon Tyne, UK

Introduction Advance care planning (ACP) allows people to record their wishes about future health care choices before their capacity is lost; in dementia, where capacity loss is inevitable, ACP has the potential to facilitate a person-centred care approach to care. This study explored the views of both professionals and people with dementia and their families about how, and if, ACP is implemented in dementia.

Method Focus groups ( $n=14)$ and one-to-one interviews ( $n=17)$ were used to explore the views of a wide range of professionals (health and social care, advocacy and legal services) involved in dementia care; $1-1$ interviews were carried out with people with dementia $(n=17)$ and their main carers $(n=29)$.

Results ACP is not routinely integrated into dementia services although some examples of good practice exist. Contrary to guidance, ACP discussions often occur late in the illness, with subsequent proxy decision-making. Professionals find ACP challenging; there appear to be additional difficulties in dementia around responsibility, assessment of capacity and legal dilemmas. People with dementia and their families do plan ahead in many areas for example, making wills and lasting power of attorney for finance. However, making plans for future healthcare appears more difficult due to a preference to 'take one day at a time'.

Conclusions ACP offers opportunities for people to plan ahead but is currently not a routine part of dementia care. Additional training for professionals may help overcome some of the barriers and hence support the people with dementia and their families who do wish to undertake this. 